\title{
Improvement of Scanning Procedure for 4D-X-ray Phase Tomography
}

\author{
Masato Hoshino ${ }^{1},{ }^{*}$, Kentaro Uesugi ${ }^{1}$, Naoto Yagi ${ }^{1}$ and Takuro Tsukube ${ }^{2}$
}

1. Japan Synchrotron Radiation Research Institute, Sayo, Hyogo, Japan

2. Japanese Red Cross Kobe Hospital, Kobe, Hyogo, Japan

* hoshino@spring8.or.jp

X-ray phase tomography is a powerful tool to measure biological soft tissues with much higher image contrast compared to the absorption-based imaging [1]. Phase information obtained from phase tomography can be treated as a density map in the hard X-ray region. So, this technique has a capability to analyze the structural and functional information from small difference and change of the density. We have developed X-ray phase tomography using a grating interferometer for the application to biological samples such as eye lenses, hearts of human infant and aortic wall and so on. More recently, we have demonstrated dynamic X-ray phase tomography so called 4D-phase tomography to discuss the dynamic phenomena of biological samples quantitatively [2]. A biological sample under continuous oscillation was observed with synchronized phase measurement using a phase stepping method. In that case, however, a frame rate of 4D-phase tomography, which was one of important parameters in dynamic measurement, was restricted by the scanning procedure. In this study, the scanning method was improved to achieve higher frame rate in the 4D measurement and more flexible measurement for dynamic applications.

4D X-ray phase tomography using a grating interferometer was developed at BL20B2 in SPring- 8 . The interferometer was composed of a phase grating (G1) and an absorption grating (G2) fabricated by NTTAT. The grating pitch of G1 and G2 was $2.6 \mu \mathrm{m}$. The X-ray energy was set to $20 \mathrm{keV}$. The $3^{\text {rd }}$ order fractional Talbot distance was used. Visibility of Moiré fringes was $26 \%$. G2 was set on the Piezo stage (P-733.2DD, PI) for phase stepping. An X-ray imaging detector was composed of a beam monitor (AA60, Hamamatsu Photonics) and sCMOS camera (ORCA Flash4.0, Hamamatsu Photonics). The effective pixel size was $7.8 \mu \mathrm{m}$. A sample was measured in the specially designed container filled with normal saline. The detailed of the sample stage is described in elsewhere [2]. In the improved system, a load cell (50N in capacity, TOYO Sokki) was installed on the sample stage to measure the load caused by stretching or compressing during a tomographic measurement.

A schematic drawing of the improved scanning method is shown in Fig.1. In the previous method, G2 set on the Piezo stage was scanned with sequential triangle-shaped analog signals from a waveform generator, which produced sequential round-trip motion of G2. In that situation, half period of the triangular wave was used for single phase stepping. On the other hand, sawtooth-shaped signal is used in the improve method. Two sawtooth waves are included in a single projection and a single periodic motion of a sample. As shown in the bottom of Fig.1, $N$ images are acquired with a fixed interval in the single sawtooth wave. Slope of the sawtooth wave is determined by the amount of voltage required for single step in $P$-step fringe scan and the interval of image acquisition. Usually $P=5$ step fringe scan is used in our system. Sequential acquisition of images is based on the assumption that G1 and G2 have a completely periodic pattern. Then, $N-P+1$ phase images are created in the single sawtooth wave. This mean that apparent frame rate of phase images is determined by the frame rate of image acquisition while $P$ sequential images are required for phase retrieval. Sawtooth waves and train of trigger signals are generated from the waveform generator where the generation of these signals is synchronized with the rotational position of the sample for tomography. 
As a demonstration of 4D X-ray phase tomography using the improved scanning method, fresh chordae tendineae extracted from a pig heart was measured. To keep freshness of the sample, the temperature of normal saline in the container was kept below 7 degrees Celsius. The chordae tendineae installed on the sample stage was stretched and released with a continuous oscillation of $0.5 \mathrm{~Hz}$ during a phase tomographic measurement. The amount of stretch was $400 \mu \mathrm{m}$ where a mechanical load caused by stretching was approximately $0.8 \mathrm{~N}$. In this measurement, projection images were acquired with a frame rate of $20 \mathrm{~Hz}$ (40msec in exposure) during a single sawtooth wave. Therefore, apparent frame rate of $20 \mathrm{~Hz}$ is expected in 4D phase tomography. X-ray phase tomographic images of chordae tendineae at the released (A) and stretched (B) conditions are shown in Fig. 2(a). The load applied to the sample and change of the cross section obtained from tomography during stretching and releasing are shown in Fig. 2(b) and 2(c), respectively. Cross section was determined by simple thresholding based on the density. In this case, the threshold was set to $1.018 \mathrm{~g} / \mathrm{cm}^{3}$. This result demonstrated that the improved technique had a capability to trace the detail of deformation process in the biological soft tissues during continuous oscillation.

In our previous scanning procedure, the frame rate of X-ray phase tomographic image was $5.7 \mathrm{~Hz}$. In the improved method, the apparent frame rate of $20 \mathrm{~Hz}$ (up to $40 \mathrm{~Hz}$ ) was demonstrated. On the other hand, a fine scan was also demonstrated with lower frame rate by increasing an exposure time. The frame rate of 4D-phase tomography could be easily changed according to nature and status of the sample and the purpose of the dynamic measurement. In addition, the proposed method may be applicable to more complex situations by using single sawtooth wave for single projection if dynamic process of the sample can be treated as a periodic motion [3].

[1] A. Momose, T. Takeda, Y. Itai and K. Hirano, Nature Medicine, 2 (1996) 473.

[2] M. Hoshino, K. Uesugi and N. Yagi, Rev. Sci. Instrum. 87 (2016) 093705.

[3] This work was made with an approval of SPring-8 Program Committee (2015B1508, 2016A1165, 2016B1293, 2017A1371, 2017B1344, 2017B1163). This work was partially supported by JST-ERATO (JPMJER1403).

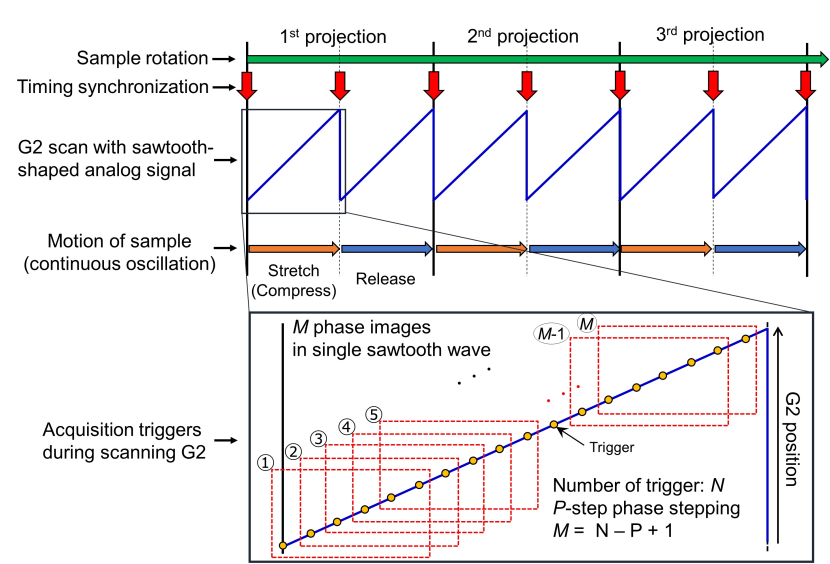

Figure. 1. Improved scanning procedure for Xray phase tomography with continuous sample oscillation.
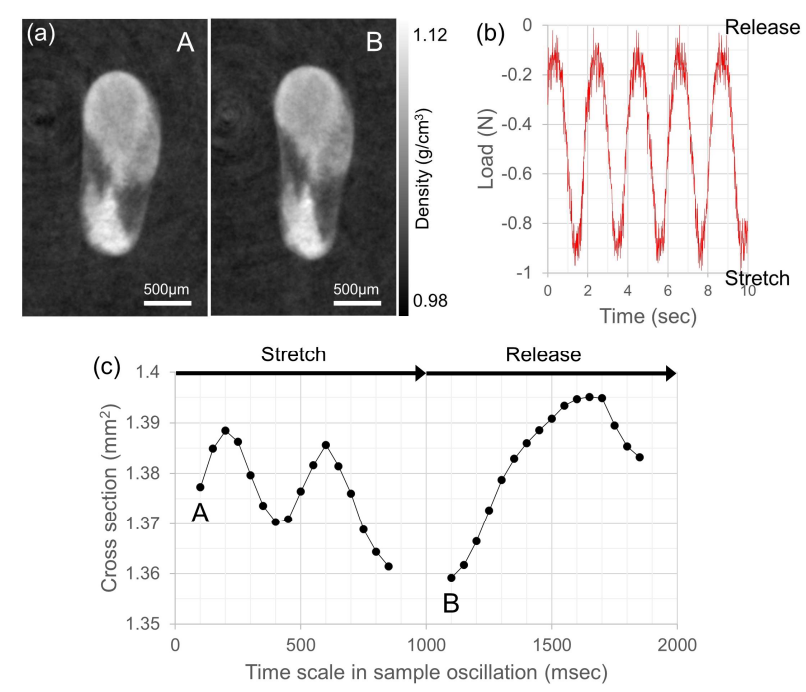

Figure. 2. (a) X-ray phase tomographic images of chordae tendineae at (A) released and (B) stretched conditions. (b) Load applied to sample during continuous oscillation. (c) Cross section of chordae tendineae during stretching and releasing. 